\title{
HOMOGENIZATION OF PERIODIC NON SELF-ADJOINT PROBLEMS WITH LARGE DRIFT AND POTENTIAL
}

\author{
GrÉgoire Allaire $^{1}$ AND RAfAel OrIVE ${ }^{2}$
}

\begin{abstract}
We consider the homogenization of both the parabolic and eigenvalue problems for a singularly perturbed convection-diffusion equation in a periodic medium. All coefficients of the equation may vary both on the macroscopic scale and on the periodic microscopic scale. Denoting by $\varepsilon$ the period, the potential or zero-order term is scaled as $\varepsilon^{-2}$ and the drift or first-order term is scaled as $\varepsilon^{-1}$. Under a structural hypothesis on the first cell eigenvalue, which is assumed to admit a unique minimum in the domain with non-degenerate quadratic behavior, we prove an exponential localization at this minimum point. The homogenized problem features a diffusion equation with quadratic potential in the whole space.
\end{abstract}

Mathematics Subject Classification. 35B27, 35K57, 35P15, 74Q10.

Received July 11, 2005. Revised June 29, 2006.

Published online July 20, 2007.

\section{INTRODUCTION}

We study problems of homogenization associated to the following singularly perturbed second order non self-adjoint elliptic operator with locally periodic coefficients

$$
\mathcal{A}^{\varepsilon} \equiv-\operatorname{div}(A(x, x / \varepsilon) \nabla)+\varepsilon^{-1} b(x, x / \varepsilon) \cdot \nabla+\varepsilon^{-2} c(x, x / \varepsilon),
$$

where the coefficients $A(x, y)=\left\{a_{i j}(x, y)\right\}_{1 \leq i, j \leq N}, b(x, y)=\left\{b_{i}(x, y)\right\}_{1 \leq i \leq N}, c(x, y)$ are real and bounded functions defined for $x \in \Omega \subset \mathbb{R}^{N}$ and $y \in \mathbb{T}^{N}$ (the unit torus). In other words, the coefficients are periodic of period $(0,1)^{N}$ with respect to the variable $y$. Furthermore, the matrix $A(x, y)$ is possibly non-symmetric, uniformly positive definite, while the vector $b(x, y)$ and the scalar potential $c(x, y)$ do not satisfy any further assumption (in particular $c$ is not necessarily non-negative and $b$ is not divergence-free nor of zero mean value). We study the asymptotic behavior, as $\varepsilon$ goes to zero, of the following parabolic problem

$$
\left\{\begin{array}{l}
\frac{\partial u_{\varepsilon}}{\partial t}+\mathcal{A}^{\varepsilon} u_{\varepsilon}=0 \quad \text { in }(0, T) \times \Omega \\
u_{\varepsilon}(t, x)=0 \quad(t, x) \in(0, T) \times \partial \Omega, \quad u_{\varepsilon}(0, x)=u_{\varepsilon}^{0}(x) \quad \text { in } \Omega
\end{array}\right.
$$

\footnotetext{
Keywords and phrases. Homogenization, non self-adjoint operators, convection-diffusion, periodic medium.

1 Centre de Mathématiques Appliquées, École Polytechnique, 91128 Palaiseau Cedex, Paris, France; gregoire.allaire@polytechnique.fr

2 Departamento de Matemáticas, Universidad Autónoma de Madrid, 28049 Madrid, Spain; rafael.orive@uam.es
}

(C) EDP Sciences, SMAI 2007 
and of the eigenvalue problem

$$
\mathcal{A}^{\varepsilon} p^{\varepsilon}=\lambda^{\varepsilon} p^{\varepsilon} \quad \text { in } \Omega, \quad p^{\varepsilon}=0 \quad \text { on } \partial \Omega .
$$

The novelty in the present work is that (1.1) is a non self-adjoint operator. The case of self-adjoint operators was previously analyzed in $[2,7,8,15]$.

In order to homogenize both (1.2) and (1.3), the main idea is to use an auxiliary non self-adjoint eigenvalue problem in the space of periodic functions (see Sect. 2). It is called exponential spectral problems (see $[6,12,13]$ ) and it is similar to the well-known Bloch spectral problem.

Our main assumption is that the first exponential eigenvalue has a single minimum at a point $x_{0} \in \Omega$ with a non-degenerate quadratic behavior near $x_{0}$ (see Sect. 2). Then, we prove that the first eigenfunction of (1.3), is approximately given as the product of an oscillating function (with period $\varepsilon$ ) and an homogenized eigenfunction concentrating exponentially at $x_{0}$ (with characteristic lengthscale $\sqrt{\varepsilon}$ ). The homogenized problem is posed on the whole space $\mathbb{R}^{N}$ with a quadratic potential linked to the Hessian of the first exponential eigenvalue (see Th. 3.5). We also prove a similar result for the parabolic equation (1.2) if the initial data are well prepared (see Th. 3.1). In other words, we build a sequence of approximate solutions of (1.2) which are defined as the product of the same oscillating function (with period $\varepsilon$ ) and of the solution of a parabolic homogenized equation, concentrating exponentially at $x_{0}$ with characteristic lengthscale $\sqrt{\varepsilon}$.

There are several motivations for studying the homogenization of (1.2) and (1.3). The first problem can be seen as a convection-diffusion-reaction equation. In particular, in the limit case of indicator-type potentials (i.e. $c=+\infty$ in a subdomain of $\mathbb{T}^{N}$ and $c=0$ elsewhere), we recover perforated domains with periodic holes supporting Dirichlet boundary conditions. In such a case the term of order $\varepsilon^{-2}$ disappears from the operator (1.1) although there is still a singular perturbation due to the presence of Dirichlet holes. This is a typical model of convection-diffusion in porous media. The self-adjoint case (for the spectral problem) was studied in [21].

The ground state asymptotic (i.e., characterizing the limit of the first eigenvalue and eigenfunction as $\varepsilon$ goes to 0 ) plays an important role when studying the long time behavior of solutions of the corresponding parabolic equation. Namely, the first eigenvalue governs the rate of decay (or growth) of solutions while the limit profile of the solutions is determined by the first eigenfunction. Another motivation for studying the eigenvalue problem is the modelling of the so-called criticality problem for the neutron diffusion equation (which allows to compute the power distribution in a nuclear reactor core, see e.g., [3]).

Apart from the already quoted papers $[2,7,8]$, there are many other related works. For example, when the coefficients are not rapidly oscillating (i.e. they depend on $x$ and not on $y$ ), the study of (1.1) is a problem of singular perturbation without homogenization [17]. The result of [17] is that, if $c(x)$ has a unique global minimum point $x_{0} \in \Omega$ then the first eigenfunction $p_{1}^{\varepsilon}(x)$ is exponentially small everywhere except at $x_{0}$ and the logarithmic asymptotic behavior of $p_{1}^{\varepsilon}$ is given. A similar logarithmic asymptotic of the ground state for an operator with locally periodic coefficients was obtained in [18]. On the other hand, when the coefficients are purely periodically oscillating functions (i.e. they depend on $y$ but not on $x$ ), the homogenization of (1.2) and (1.3) is also quite well understood, and more precise results are obtained in $[3,5,13,14]$. In the case $b \equiv 0$ and $c \equiv 0$ we also refer to [20]. Similar problems, with a different scaling, are also studied using probabilistic tools in $[10,11]$.

The rest of the paper is organized as follows: in Section 2 we present the non-self adjoint exponential eigenvalue problem and our assumptions on the cell ground state. In Section 3 we state our main homogenization results. In Sections 4 and 5 we prove the convergence of the homogenization process for the parabolic problem and the eigenvalue problem, respectively.

\section{Notations And Assumptions}

In order to formulate our assumptions on the operator $\mathcal{A}^{\varepsilon}$ we introduce an auxiliary eigenvalue problem: the so-called exponential cell eigenproblem. Following e.g. $[6,12,13]$, for any parameter vector $\theta \in \mathbb{R}^{N}$ and for any 
point $x \in \Omega$, we introduce the following $\theta$-exponential periodic problem: find $\left(\lambda(x, \theta), \psi_{x, \theta}\right)$ the first eigenpair of

$$
\left\{\begin{array}{l}
-\operatorname{div}_{y}\left(A(x, y) \nabla_{y} \psi_{x, \theta}\right)+b(x, y) \cdot \nabla_{y} \psi_{x, \theta}+c(x, y) \psi_{x, \theta}=\lambda(x, \theta) \psi_{x, \theta} \\
y \rightarrow \psi_{x, \theta}(y) e^{-2 \pi \theta \cdot y}(0,1)^{N} \text {-periodic. }
\end{array}\right.
$$

Here and in the sequel we denote by $\nabla_{y}$ the derivative in the oscillating variable and by $\nabla_{x}$ the derivative with respect to the macroscopic variable. Analogously, denoting by $A^{*}$ the adjoint matrix of $A$, we consider the adjoint problem: find $\left(\lambda^{*}(x, \theta), \psi_{x, \theta}^{*}\right)$ the first eigenpair of

$$
\left\{\begin{array}{l}
-\operatorname{div}_{y}\left(A^{*}(x, y) \nabla_{y} \psi_{x, \theta}^{*}\right)-\operatorname{div}_{y}\left(b(x, y) \psi_{x, \theta}^{*}\right)+c(x, y) \psi_{x, \theta}^{*}=\lambda^{*}(x, \theta) \psi_{x, \theta}^{*}, \\
y \rightarrow \psi_{x, \theta}^{*}(y) e^{2 \pi \theta \cdot y}(0,1)^{N} \text {-periodic. }
\end{array}\right.
$$

Of course, in (2.1) and (2.2), $\psi_{x, \theta}$ and $\psi_{x, \theta}^{*}$ are functions of the variable $y$ ( $x \in \Omega$ is just a parameter, like $\theta$ ). Equations (2.1) and (2.2) are posed on the whole space $\mathbb{R}^{N}$, but, due to the "exponential periodic" boundary condition, they can be restricted to the unit cell $(0,1)^{N}$. In order to find a convenient formulation of the boundary condition we change the unknown as

$$
\psi_{x, \theta}(y)=\mathrm{e}^{2 \pi \theta \cdot y} \phi_{x, \theta}(y) \quad \text { and } \quad \psi_{x, \theta}^{*}(y)=\mathrm{e}^{-2 \pi \theta \cdot y} \phi_{x, \theta}^{*}(y),
$$

where $\phi_{x, \theta}$ and $\phi_{x, \theta}^{*}$ are now $(0,1)^{N}$-periodic functions. This allows us to rewrite an equivalent form of $(2.1)$ on the unit torus $\mathbb{T}^{N}$ (a compact manifold)

$$
-\left(\operatorname{div}_{y}+2 \pi \theta\right)\left(A(x, y)\left(\nabla_{y}+2 \pi \theta\right) \phi_{x, \theta}\right)+b(x, y)\left(\nabla_{y}+2 \pi \theta\right) \phi_{x, \theta}+c(x, y) \phi_{x, \theta}=\lambda(x, \theta) \phi_{x, \theta},
$$

and similarly for the adjoint problem (2.2). Remark that there is no $i$ or imaginary numbers in (2.3) which is the main difference with the Bloch cell problems. In particular (2.3) is not self-adjoint, even if $A$ is symmetric and $b=0$. However, since (2.3) is a real-valued problem and the corresponding Green operator is compact, one can apply the Krein-Rutman theorem which is at the basis of the next result. By an obvious generalization of Lemma 3 of [13], we get the following result:

Lemma 2.1. For all $\theta \in \mathbb{R}^{N}$ and $x \in \Omega$, there exist a first eigenvalue and eigenfunction $\psi_{x, \theta}$ to (2.1) and $\psi_{x, \theta}^{*}$ to (2.2). The first eigenvalue of (2.1) and (2.2) is the same, i.e. $\lambda(x, \theta)=\lambda^{*}(x, \theta)$. It is real, of smallest modulus among all eigenvalues and of geometric and algebraic multiplicity equal to one. The first eigenfunctions $\psi_{x, \theta}$ and $\psi_{x, \theta}^{*}$ can be chosen positive in $\mathbb{T}^{N}$.

Furthermore, for all $x \in \Omega$, the function $\theta \rightarrow \lambda(x, \theta)$ is concave from $\mathbb{R}^{N}$ into $\mathbb{R}$ and admits a maximum $\lambda_{\infty}(x)$ which is obtained for a unique $\theta=\theta_{\infty}(x)$. Denoting by $\psi_{\infty}(x, y)$ the corresponding eigenfunction $\psi_{x, \theta_{\infty}(x)}(y)$ (and similarly for the adjoint), it is characterized by the following relation

$$
\int_{\mathbb{T}^{N}} \beta(x, y) \mathrm{d} y=0,
$$

where the divergence-free vector $\beta$ is defined by

$$
\beta(x, y)=\psi_{\infty}^{*} \psi_{\infty} b(x, y)+\psi_{\infty} A^{*} \nabla_{y} \psi_{\infty}^{*}(x, y)-\psi_{\infty}^{*} A \nabla_{y} \psi_{\infty}(x, y) .
$$

Remark 2.2. The characterization (2.4) is actually equivalent to the optimality condition $\nabla_{\theta} \lambda\left(x, \theta_{\infty}(x)\right)=0$ [13]. In the sequel a case of special interest will be $\theta_{\infty}(x)=0$ (see Rem. 3.2). It is clear that if the operator (1.1) is self-adjoint, i.e. if the matrix $A$ is symmetric and the vector $b$ is equal to 0 , then $\psi_{x, 0}^{*}=\psi_{x, 0}$ which implies $\beta \equiv 0$ and $\theta_{\infty}(x)=0$. Another sufficient condition to have $\theta_{\infty}(x)=0$ is the following central symmetry with respect to the center of the unit cell $(-1 / 2 ; 1 / 2)^{N}$. If we assume that $A(-y)=A(y), c(-y)=c(y)$ and $b(-y)=-b(y)$, then $\psi_{x, 0}^{*}(-y)=\psi_{x, 0}^{*}(y)$ and $\psi_{x, 0}(-y)=\psi_{x, 0}(y)$, so that $\beta$ is an odd function of $y$ and condition (2.4) is satisfied for $\theta_{\infty}(x)=0$. 
Remark 2.3. Since the eigenfunctions are positive and continuous on $\mathbb{T}^{N}$, there exists a positive constant $C$ (depending on $x$ and $\theta$ ) such that $\psi_{x, \theta}(y) \geq C>0$ and $\psi_{x, \theta}^{*}(y) \geq C>0$ uniformly in $y \in \mathbb{T}^{N}$. As is usual we normalize the eigenfunctions by imposing

$$
\int_{\mathbb{T}^{N}}\left|\phi_{x, \theta}(y)\right|^{2} \mathrm{~d} y=1 \quad \text { and } \quad \int_{\mathbb{T}^{N}} \phi_{x, \theta}(y) \phi_{x, \theta}^{*}(y) \mathrm{d} y=\int_{\mathbb{T}^{N}} \psi_{x, \theta}(y) \psi_{x, \theta}^{*}(y) \mathrm{d} y=1 .
$$

A consequence of the simplicity of $\lambda(x, \theta)$ and of the above normalization is that the eigenvalue and eigenfunctions have the same differentiability property as the coefficients with respect to $x$.

Our main assumptions on $\lambda_{\infty}(x)=\lambda\left(x, \theta_{\infty}(x)\right)$ with respect to its dependence on $x$ are:

Hypothesis H1. The function $x \rightarrow \lambda_{\infty}(x)$ has a unique global minimum point $x_{0}$ in the interior of $\Omega$.

Hypothesis H2. The coefficients $a_{i j}(x, y), b_{j}(x, y)$ and $c(x, y)$ are of class $C^{2}$ in $\bar{\Omega} \times \mathbb{T}^{N}$, and the Taylor series for $\lambda_{\infty}(x)$ about $x_{0}$ has non-degenerate (positive definite) quadratic form

$$
\lambda_{\infty}(x)=\lambda_{\infty}\left(x_{0}\right)+\Lambda_{i j}\left(x-x_{0}\right)_{i}\left(x-x_{0}\right)_{j}+o\left(\left|x-x_{0}\right|^{2}\right), \quad \Lambda_{i j} \xi_{i} \xi_{j} \geq c|\xi|^{2} \quad(c>0),
$$

for any vector $\xi \in \mathbb{R}^{N}$, where

$$
\Lambda_{i j} \equiv \frac{1}{2} \frac{\partial^{2} \lambda_{\infty}}{\partial x_{i} \partial x_{j}}\left(x_{0}\right)
$$

Without loss of generality we shall assume in the sequel that $x_{0}=0$ and we denote $\psi_{\infty}(y) \equiv \psi_{\infty}(0, y)$, $\theta_{\infty}=\theta_{\infty}(0)$ and $\lambda_{\infty}=\lambda_{\infty}(0)$.

Remark 2.4. Hypothesis $\mathbf{H 1}$ ensures the concentration of $\psi_{\infty}(x, x / \varepsilon)$ in the neighborhood of $x_{0}$ while Hypothesis $\mathbf{H 2}$ allows to characterize, in the vicinity of $x_{0}$, the asymptotic behavior of its profile.

Notations. For any function $\phi(x, y)$ defined on $\mathbb{R}^{N} \times \mathbb{T}^{N}$, we denote by $\phi^{\varepsilon}$ the function $\phi(x, x / \varepsilon)$. For a variable $z \in \mathbb{R}^{N}$, we denote by $L^{2}\left(\mathbb{R}^{+} ; L^{2}\left(\mathbb{R}^{N} ;|z|^{2}\right)\right)$ the space defined by

$$
L^{2}\left(\mathbb{R}^{+} ; L^{2}\left(\mathbb{R}^{N} ;|z|^{2}\right)\right)=\left\{v(t, z) \in L^{2}\left(\mathbb{R}^{+} \times \mathbb{R}^{N}\right) \text { such that }\||z| v\|_{L^{2}\left(\mathbb{R}^{+} \times \mathbb{R}^{N}\right)}<\infty\right\} .
$$

\section{Main Results}

Let $\Omega \subset \mathbb{R}^{N}$ be an open set (bounded or not). Let $0<T<+\infty$ be a final time. We first consider the following parabolic problem

$$
\left\{\begin{array}{l}
\frac{\partial u_{\varepsilon}}{\partial t}+\mathcal{A}^{\varepsilon} u_{\varepsilon}=0 \quad \text { in }(0, T) \times \Omega, \\
u_{\varepsilon}(t, x)=0 \quad(x, t) \in \partial \Omega \times(0, T), \\
u_{\varepsilon}(0, x)=u_{\varepsilon}^{0}(x) \quad \text { in } \Omega,
\end{array}\right.
$$

with $\mathcal{A}^{\varepsilon}$ defined in (1.1). Hille-Yosida-Phillip's theorem guarantees that (3.1) generates a semigroup of contractions. Thus, for any initial data $u_{\varepsilon}^{0}$ which belongs to $L^{2}(\Omega),(3.1)$ has a unique weak solution $u_{\varepsilon}=u_{\varepsilon}(x, t)$ such that $u_{\varepsilon} \in C\left([0, T] ; L^{2}(\Omega)\right) \cap L^{2}\left((0, T) ; H_{0}^{1}(\Omega)\right)$.

Theorem 3.1. Assume $\mathrm{H} 1$ and $\mathrm{H} 2$ and that the initial data $u_{\varepsilon}^{0} \in L^{2}(\Omega)$ is of the form

$$
u_{\varepsilon}^{0}(x)=\psi_{\infty}(x, x / \varepsilon) w^{0}(x / \sqrt{\varepsilon}),
$$

with $w^{0} \in L^{2}\left(\mathbb{R}^{N}\right)$. The solution of $(3.1)$ can be written as

$$
u_{\varepsilon}(t, x)=\mathrm{e}^{-\frac{\lambda_{\infty}}{\varepsilon^{2}} t} w_{\varepsilon}(t / \varepsilon, x / \sqrt{\varepsilon}) \psi_{\infty}(x, x / \varepsilon),
$$


where $w^{\varepsilon}(s, z)$ is a function which converges weakly in $L^{2}\left(\mathbb{R}^{+} ; H^{1}\left(\mathbb{R}^{N}\right)\right) \cap L^{\infty}\left(\mathbb{R}^{+} ; L^{2}\left(\mathbb{R}^{N}\right)\right) \cap L^{2}\left(\mathbb{R}^{+} ; L^{2}\left(\mathbb{R}^{N} ;|z|^{2}\right)\right)$ and strongly in $L^{2}\left([0, S] ; L^{2}\left(\mathbb{R}^{N}\right)\right.$ ) (for any finite time $S$ ) to the solution $w$ of the homogenized problem

$$
\left\{\begin{array}{l}
\frac{\partial w}{\partial t}-\operatorname{div}\left(A^{\mathrm{eff}} \nabla w\right)+\left(\Lambda_{i j} z_{i} z_{j}+d^{\mathrm{eff}}\right) w=0 \quad \text { in } \mathbb{R}^{+} \times \mathbb{R}^{N} \\
w(0, z)=w^{0}(z) \quad \text { in } \mathbb{R}^{N}
\end{array}\right.
$$

with

$$
d^{\mathrm{eff}}=\int_{\mathbb{T}^{N}}\left(\psi_{\infty}^{*} b \nabla_{x} \psi_{\infty}-\psi_{\infty}^{*} \operatorname{div}_{y}\left(A \nabla_{x} \psi_{\infty}\right)-\psi_{\infty}^{*} \operatorname{div}_{x}\left(A \nabla_{y} \psi_{\infty}\right)\right)(0, y) \mathrm{d} y,
$$

and the matrix $A^{\mathrm{eff}}=\left\{a_{i j}^{\mathrm{eff}}\right\}_{1 \leq i, j \leq N}$ is given by

$$
a_{i j}^{\mathrm{eff}}=\int_{\mathbb{T}^{N}} \psi_{\infty} \psi_{\infty}^{*} A(0, y) \nabla\left(\chi_{j}+y_{j}\right) \cdot \nabla\left(\chi_{i}+y_{i}\right) \mathrm{d} y
$$

where the functions $\chi_{i}$ are defined for $i=1, \ldots, N$ by:

$$
\left\{\begin{array}{l}
-\operatorname{div}_{y}\left(\psi_{\infty} \psi_{\infty}^{*} A(0, y) \nabla_{y}\left(\chi_{i}+y_{i}\right)\right)+\beta(0, y) \cdot \nabla_{y}\left(\chi_{i}+y_{i}\right)=0 \quad \text { in } \mathbb{T}^{N}, \\
y \rightarrow \chi_{i}(y) \quad \mathbb{T}^{N} \text {-periodic, }
\end{array}\right.
$$

with the vector $\beta$ defined in (2.5).

Remark 3.2. The initial data (3.2) is well prepared. Indeed, recall that

$$
\psi_{\infty}(x, x / \varepsilon)=\mathrm{e}^{2 \pi \theta_{\infty} \cdot x / \varepsilon} \phi_{\infty}(x, x / \varepsilon),
$$

where $\phi_{\infty}(x, y)$ is $(0,1)^{N}$-periodic in the variable $y$. This implies that $\psi_{\infty}$ has an exponential behavior, except if $\theta_{\infty}=0$. In the special case $\theta_{\infty}=0$, the initial data is uniformly bounded. Furthermore, there is no need to have well prepared initial data in such a case since for any sequence $u_{\varepsilon}^{0}$, uniformly bounded in $L^{2}(\Omega)$, one can extract a subsequence which has the same limit behavior (in the sense of two-scale convergence) as (3.2).

Remark 3.3. The periodic cell problems (3.7) are well posed for $1 \leq i \leq N$. Indeed, by Fredholm's alternative, there exists a unique solution (up to an additive constant) of (3.7) if and only if

$$
\int_{\mathbb{T}^{N}} \beta(0, y) \cdot \nabla_{y}\left(\chi_{i}+y_{i}\right) \mathrm{d} y=0 .
$$

This is satisfied since $\beta$ defined in (2.5) is divergence free and satisfies condition (2.4).

Remark 3.4. Theorem 2 still holds true if we add a source term of order $\varepsilon^{-1}$. Indeed, we can add to (3.1) the following source term

$$
f_{\varepsilon}(t, x)=\frac{1}{\varepsilon} \mathrm{e}^{-\frac{\lambda \infty}{\varepsilon^{2}} t} f(t / \varepsilon, x, x / \sqrt{\varepsilon}, x / \varepsilon),
$$

where $f(t, x, z, y) \in L^{2}\left(\mathbb{R}^{+} \times \Omega \times \mathbb{R}^{N} \times \mathbb{T}^{N}\right)$. In such a case, the homogenized equation (3.4) has an additional source term which is $f^{\text {eff }}(t, z)$ defined by

$$
f^{\mathrm{eff}}(t, z)=\int_{\mathbb{T}^{N}} f(t, 0, z, y) \psi_{\infty}^{*}(0, y) \mathrm{d} y
$$


Also, we can add to equation (3.1) a non-linear term $\varepsilon^{-1} g\left(x, x / \sqrt{\varepsilon}, x / \varepsilon, u_{\varepsilon}\right)$ where $g(x, z, y, \xi)$ is a continuous function, homogeneous of degree one and uniformly Lipschitz with respect to the variable $\xi$. Hence, the homogenized equation (3.4) has an additional non-linear term $g^{\text {eff }}(z, w)$ defined by

$$
g^{\mathrm{eff}}(z, w)=\int_{\mathbb{T}^{N}} g\left(0, z, y, \psi_{\infty}(0, y) w\right) \psi_{\infty}^{*}(0, y) \mathrm{d} y
$$

Next, for a bounded open set $\Omega$, we consider the following eigenvalue problem

$$
\left\{\begin{array}{l}
\mathcal{A}^{\varepsilon} p^{\varepsilon}=\lambda^{\varepsilon} p^{\varepsilon} \quad \text { in } \Omega \\
p^{\varepsilon}=0 \quad \text { on } \partial \Omega
\end{array}\right.
$$

with $\mathcal{A}^{\varepsilon}$ defined in (1.1). As is well known, for each fixed $\varepsilon>0$ this operator has compact inverse in $L^{2}(\Omega)$ (possibly non self-adjoint) and satisfies the maximum principle. Thus, by the Krein-Rutman theorem, we have a first eigenvalue $\lambda_{1}^{\varepsilon}$ of multiplicity one and the corresponding eigenfunction $p_{1}^{\varepsilon}$ can be chosen positive in $\Omega$.

Theorem 3.5. Under assumption $\mathrm{H} 1$ and $\mathrm{H} 2$ we have

$$
\lambda_{1}^{\varepsilon}=\lambda_{\infty}+\varepsilon \mu_{1}+o(\varepsilon) \quad \text { with } \lim _{\varepsilon \rightarrow 0} \frac{o(\varepsilon)}{\varepsilon}=0
$$

and the corresponding eigenvector $p_{1}^{\varepsilon}$ admits the representation

$$
p_{1}^{\varepsilon}(x)=q_{1}^{\varepsilon}(x / \sqrt{\varepsilon}) \psi_{\infty}(x, x / \varepsilon),
$$

where $q_{1}^{\varepsilon}(z) \in H^{1}\left(\mathbb{R}^{N}\right)$, up to a renormalization, converges, as $\varepsilon$ goes to zero, to a limit $q_{1}(z)$ which is the first eigenfunction associated to the first eigenvalue $\mu_{1}$ of the homogenized spectral problem

$$
-\operatorname{div}\left(A^{\mathrm{eff}} \nabla q\right)+\left(\Lambda_{i j} z_{i} z_{j}+d^{\mathrm{eff}}\right) q=\mu q \quad \text { in } \mathbb{R}^{N}
$$

with $d^{\mathrm{eff}}$ and $A^{\mathrm{eff}}$ defined by (3.5) and (3.6), respectively.

Remark 3.6. The spectral problem (3.10) has a purely punctual spectrum since $\Lambda_{i j} z_{i} z_{j}$ is a non-degenerate quadratic potential. This is the so-called harmonic oscillator problem (see e.g. [7]).

\section{Parabolic problem}

This section is devoted to the proof of Theorem 3.1. We introduce a new unknown in the spirit of $[8,13]$,

$$
v_{\varepsilon}(t, x)=\mathrm{e}^{\frac{\lambda_{\infty}}{\varepsilon^{2}} t} \frac{u_{\varepsilon}(t, x)}{\psi_{\infty}(x, x / \varepsilon)}
$$

which is well defined since $\psi_{\infty}$ is positive as already mentioned in Remark 2.3. We replace $u_{\varepsilon}$ by $v_{\varepsilon}$ in (3.1) and we recall that $\psi_{\infty}(x, y)$ and $\psi_{\infty}^{*}(x, y)$ are the first eigenfunction of $(2.1)$ and $(2.2)$, respectively. After some algebra we obtain that $(3.1)$ is equivalent to

$$
\left\{\begin{array}{l}
\varepsilon \sigma^{\varepsilon} \frac{\partial v_{\varepsilon}}{\partial t}-\varepsilon \operatorname{div}\left(\alpha^{\varepsilon} \nabla v_{\varepsilon}\right)+\beta^{\varepsilon} \cdot \nabla v_{\varepsilon} \\
\quad+\sigma^{\varepsilon} \frac{\lambda_{\infty}(x)-\lambda_{\infty}}{\varepsilon} v_{\varepsilon}+\varepsilon B^{\varepsilon} \cdot \nabla v_{\varepsilon}+\Sigma^{\varepsilon} v_{\varepsilon}=0 \quad \text { in } \mathbb{R}^{+} \times \Omega \\
v_{\varepsilon}(t, x)=0 \quad(t, x) \stackrel{\mathbb{R}^{+} \times \partial \Omega}{u_{\infty}(x, x / \varepsilon)} \quad \text { in } \Omega \\
v_{\varepsilon}(0, x)=\frac{u_{\varepsilon}^{0}(x)}{\psi_{\infty}(x)}
\end{array}\right.
$$


where

$$
\sigma^{\varepsilon}(x)=\psi_{\infty} \psi_{\infty}^{*}(x, x / \varepsilon), \quad \alpha^{\varepsilon}(x)=\psi_{\infty}^{*} \psi_{\infty} A(x, x / \varepsilon), \quad \beta^{\varepsilon}(x)=\beta(x, x / \varepsilon),
$$

and

$$
\begin{aligned}
& B^{\varepsilon}(x)=\psi_{\infty} A^{*} \nabla_{x} \psi_{\infty}^{*}(x, x / \varepsilon)-\psi_{\infty}^{*} A \nabla_{x} \psi_{\infty}(x, x / \varepsilon) \\
& \Sigma^{\varepsilon}(x)=\Sigma_{0}^{\varepsilon}(x)+\varepsilon \Sigma_{1}^{\varepsilon}(x)
\end{aligned}
$$

with

$$
\begin{aligned}
& \Sigma_{0}^{\varepsilon}(x)=\psi_{\infty}^{*} b \nabla_{x} \psi_{\infty}(x, x / \varepsilon)-\psi_{\infty}^{*} \operatorname{div}_{y} A \nabla_{x} \psi_{\infty}(x, x / \varepsilon)-\psi_{\infty}^{*} \operatorname{div}_{x} A \nabla_{y} \psi_{\infty}(x, x / \varepsilon), \\
& \Sigma_{1}^{\varepsilon}(x)=-\psi_{\infty}^{*} \operatorname{div}_{x} A \nabla_{x} \psi_{\infty}(x, x / \varepsilon) .
\end{aligned}
$$

Remark that $\sigma^{\varepsilon}$ converges weakly- $\star$ in $L^{\infty}\left(\mathbb{R}^{N}\right)$ to 1 because of the normalization condition (2.6), while $\Sigma_{0}^{\varepsilon}$ converges weakly- $\star$ in $L^{\infty}\left(\mathbb{R}^{N}\right)$ to $d^{\text {eff }}$ defined by (3.5).

In order to eliminate the $\varepsilon$ scaling in front of the second-order operator in (4.2) and to obtain uniform a priori estimates, we rescale the space and time variable by introducing

$$
z=\frac{x}{\sqrt{\varepsilon}} \in \Omega^{\varepsilon}=\varepsilon^{-1 / 2} \Omega, \quad s=\frac{t}{\varepsilon}
$$

and consider the function

$$
w_{\varepsilon}(s, z)=v_{\varepsilon}(t, x), \quad(s, z) \in \mathbb{R}^{+} \times \Omega^{\varepsilon} .
$$

This yields

$$
\left\{\begin{array}{l}
\widetilde{\sigma}^{\varepsilon} \frac{\partial w_{\varepsilon}}{\partial s}+\widetilde{\mathcal{A}}^{\varepsilon} w_{\varepsilon}+\widetilde{\sigma}^{\varepsilon} \frac{\lambda_{\infty}(\sqrt{\varepsilon} z)-\lambda_{\infty}}{w_{\varepsilon}} w_{\varepsilon}+\sqrt{\varepsilon} \widetilde{B}^{\varepsilon} \cdot \nabla w_{\varepsilon}+\widetilde{\Sigma}^{\varepsilon} w_{\varepsilon}=0 \quad \text { in } \mathbb{R}^{+} \times \Omega^{\varepsilon} \\
w_{\varepsilon}(s, z)=0 \quad(s, z) \in \mathbb{R}^{+} \times \partial \Omega^{\varepsilon} \\
w_{\varepsilon}(0, z)=w_{\varepsilon}^{0}(z)=\frac{u_{\varepsilon}^{0}(\sqrt{\varepsilon} z)}{\psi_{\infty}^{\varepsilon}(z)} \quad \text { in } \Omega^{\varepsilon}
\end{array}\right.
$$

where the operator $\widetilde{\mathcal{A}}^{\varepsilon}$ is defined by

$$
\widetilde{\mathcal{A}}^{\varepsilon} \equiv-\operatorname{div}\left(\widetilde{\alpha}^{\varepsilon}(z) \nabla\right)+\varepsilon^{-1 / 2} \widetilde{\beta}^{\varepsilon}(z) \cdot \nabla .
$$

In (4.7) and (4.8), we use the notation $\widetilde{\phi}^{\varepsilon}(z)=\phi(\sqrt{\varepsilon} z, z / \sqrt{\varepsilon})$ for any function $\phi(x, y)$ defined on $\mathbb{R}^{N} \times \mathbb{T}^{N}$. Now, we can obtain the following a priori estimate.

Lemma 4.1. Let $0<S<+\infty$ be a final time. Assume that $w^{\varepsilon}$ satisfies (4.7) with $w_{\varepsilon}^{0} \in L^{2}\left(\Omega^{\varepsilon}\right)$. Then,

$$
\left\|w_{\varepsilon}\right\|_{L^{\infty}\left((0, S) ; L^{2}\left(\Omega^{\varepsilon}\right)\right)}+\left\|\nabla w_{\varepsilon}\right\|_{L^{2}\left((0, S) \times \Omega^{\varepsilon}\right)^{N}}+\left\|z \mid w_{\varepsilon}\right\|_{L^{2}\left((0, S) \times \Omega^{\varepsilon}\right)} \leq C(S)\left\|w_{\varepsilon}^{0}\right\|_{L^{2}\left(\Omega^{\varepsilon}\right)},
$$

and, for any function $\varphi(s, z)$ in the space $L^{2}\left([0, S] ; H^{1}\left(\mathbb{R}^{N}\right)\right) \cap L^{2}\left([0, S] ; L^{2}\left(\mathbb{R}^{N} ;|z|^{2}\right)\right)$,

$$
\left|\int_{0}^{S} \int_{\Omega^{\varepsilon}} \frac{\partial w_{\varepsilon}}{\partial s} \varphi \mathrm{d} z \mathrm{~d} s\right| \leq C(S)\left\|w_{\varepsilon}^{0}\right\|_{L^{2}\left(\Omega^{\varepsilon}\right)}\left(\|\varphi\|_{L^{2}\left([0, S] ; H^{1}\left(\mathbb{R}^{N}\right)\right)}+\|\varphi\|_{L^{2}\left([0, S] ; L^{2}\left(\mathbb{R}^{N} ;|z|^{2}\right)\right)}\right),
$$

with $C(S)>0$ independent of $\varepsilon$. 
Proof. First, we multiply equation (4.7) by $w_{\varepsilon}$ and we integrate by parts to obtain

$$
\begin{aligned}
& \frac{1}{2} \int_{\Omega^{\varepsilon}} \widetilde{\sigma}^{\varepsilon}(z)\left|w_{\varepsilon}(S, z)\right|^{2} \mathrm{~d} z-\frac{1}{2} \int_{\Omega^{\varepsilon}} \widetilde{\sigma}^{\varepsilon}(z)\left|w_{\varepsilon}^{0}(z)\right|^{2} \mathrm{~d} z \\
& +\int_{0}^{S} \int_{\Omega^{\varepsilon}} \widetilde{\mathcal{A}}^{\varepsilon} w_{\varepsilon}(s, z) \cdot w_{\varepsilon}(s, z) \mathrm{d} z \mathrm{~d} s+\int_{0}^{S} \int_{\Omega^{\varepsilon}} \widetilde{\sigma}^{\varepsilon} \frac{\lambda_{\infty}(\sqrt{\varepsilon} z)-\lambda_{\infty}}{\varepsilon}\left|w_{\varepsilon}(s, z)\right|^{2} \mathrm{~d} z \mathrm{~d} s \\
& \quad+\sqrt{\varepsilon} \int_{0}^{S} \int_{\Omega^{\varepsilon}} \widetilde{B}^{\varepsilon}(z) \cdot \nabla w_{\varepsilon}(s, z) w_{\varepsilon}(s, z) \mathrm{d} z \mathrm{~d} s+\int_{0}^{S} \int_{\Omega^{\varepsilon}} \widetilde{\Sigma}^{\varepsilon}(z)\left|w_{\varepsilon}(s, z)\right|^{2} \mathrm{~d} z \mathrm{~d} s=0 .
\end{aligned}
$$

By virtue of our smoothness assumption on the coefficients, the vector $\widetilde{B}^{\varepsilon}$ is uniformly bounded and CauchySchwartz inequality yields

$$
\int_{0}^{S} \int_{\Omega^{\varepsilon}} \widetilde{B}^{\varepsilon}(z) \cdot \nabla w_{\varepsilon}(s, z) w_{\varepsilon}(s, z) \mathrm{d} z \mathrm{~d} s \leq c\left\|w_{\varepsilon}\right\|_{L^{2}\left((0, S) \times \Omega^{\varepsilon}\right)}\left\|\nabla w_{\varepsilon}\right\|_{L^{2}\left((0, S) \times \Omega^{\varepsilon}\right)} .
$$

Analogously, the function $\widetilde{\Sigma}_{0}^{\varepsilon}$ and $\widetilde{\Sigma}_{1}^{\varepsilon}$ are uniformly bounded. Therefore,

$$
\int_{0}^{S} \int_{\Omega^{\varepsilon}}\left|\widetilde{\Sigma}^{\varepsilon}(z)\right|\left|w_{\varepsilon}(s, z)\right|^{2} \mathrm{~d} z \mathrm{~d} s \leq c\left\|w_{\varepsilon}\right\|_{L^{2}\left((0, S) \times \Omega^{\varepsilon}\right)}^{2} .
$$

The non-self adjoint operator $\widetilde{\mathcal{A}}^{\varepsilon}$ defined in (4.8) can be partly written in divergence form. Indeed, by (4.3) we have $\widetilde{\beta}^{\varepsilon}(z)=\beta(\sqrt{\varepsilon} z, z / \sqrt{\varepsilon})$ where the vector $\beta$, defined by (2.5), has zero average in $y$ and is divergence free by virtue of Lemma 2.1. Thus there exists a skew-symmetric matrix $D(x, y)$, defined by its entries

$$
d_{i j}(x, y)=\left(-\Delta_{y}\right)^{-1}\left(\frac{\partial \beta_{i}}{\partial y_{j}}(x, y)-\frac{\partial \beta_{j}}{\partial y_{i}}(x, y)\right)
$$

such that $\operatorname{div}_{y} D=-\beta$ (see [13] if necessary) and the operator $\widetilde{\mathcal{A}}^{\varepsilon}$ can be written as

$$
\widetilde{\mathcal{A}}^{\varepsilon} \varphi=-\operatorname{div}\left(\left(\widetilde{\alpha}^{\varepsilon}(z)+\widetilde{D}^{\varepsilon}(z)\right) \nabla \varphi\right)+\sqrt{\varepsilon} \operatorname{div}_{x} \widetilde{D}^{\varepsilon}(z) \cdot \nabla \varphi
$$

The hypotheses on the coefficients imply that $\operatorname{div}_{x} \widetilde{D}^{\varepsilon}(z)$ is uniformly bounded. Thus, since $\widetilde{D}^{\varepsilon}$ is a skewsymmetric matrix and the matrix $\widetilde{\alpha}^{\varepsilon}$ defined in (4.3) is coercive, we get

$$
\int_{0}^{S} \int_{\Omega^{\varepsilon}} \widetilde{\mathcal{A}}^{\varepsilon} w_{\varepsilon}(s, z) \cdot w_{\varepsilon}(s, z) \mathrm{d} z \mathrm{~d} s \geq c\left\|\nabla w_{\varepsilon}\right\|_{L^{2}\left((0, S) \times \Omega^{\varepsilon}\right)}^{2}-c \sqrt{\varepsilon}\left\|w_{\varepsilon}\right\|_{L^{2}\left((0, S) \times \Omega^{\varepsilon}\right)}\left\|\nabla w_{\varepsilon}\right\|_{L^{2}\left((0, S) \times \Omega^{\varepsilon}\right)} .
$$

On the other hand, hypothesis $\mathrm{H} 2$ implies that there exists two positive constants $0<c_{1}<c_{2}$ such that

$$
c_{1}|z|^{2} \leq \frac{\lambda_{\infty}(\sqrt{\varepsilon} z)-\lambda_{\infty}}{\varepsilon} \leq c_{2}|z|^{2} \quad \forall z \in \Omega^{\varepsilon} .
$$


Thus, using also the fact that $C>\sigma^{\varepsilon}(z)>c>0$, we deduce

$$
\int_{\Omega^{\varepsilon}}\left|w_{\varepsilon}(S, z)\right|^{2} \mathrm{~d} z+\int_{0}^{S} \int_{\Omega^{\varepsilon}}\left|\nabla w_{\varepsilon}(s, z)\right|^{2} \mathrm{~d} z \mathrm{~d} s+\int_{0}^{S} \int_{\Omega^{\varepsilon}}|z|^{2}\left|w_{\varepsilon}(s, z)\right|^{2} \mathrm{~d} z \mathrm{~d} s \leq C \int_{\Omega^{\varepsilon}}\left|w_{\varepsilon}^{0}(z)\right|^{2} \mathrm{~d} z+C \int_{0}^{S} \int_{\Omega^{\varepsilon}}\left|w_{\varepsilon}(s, z)\right|^{2} \mathrm{~d} z \mathrm{~d} s .
$$

Thus, by Gronwall's Lemma, we immediately obtain that

$$
\int_{0}^{S} \int_{\Omega^{\varepsilon}}\left|w_{\varepsilon}(s, z)\right|^{2} \mathrm{~d} z \leq \mathrm{e}^{c S} \int_{\Omega^{\varepsilon}}\left|w_{\varepsilon}^{0}(z)\right|^{2} \mathrm{~d} z
$$

which yields estimate (4.9).

Now, we multiply equation (4.7) by $\varphi \in L^{2}\left(\mathbb{R}^{+} ; H^{1}\left(\mathbb{R}^{N}\right)\right) \cap L^{2}\left(\mathbb{R}^{+} ; L^{2}\left(\mathbb{R}^{N} ;|z|^{2}\right)\right)$. Then,

$$
\begin{aligned}
\left|\int_{0} \int_{\Omega^{\varepsilon}} \tilde{\sigma}^{\varepsilon} \frac{\partial w_{\varepsilon}}{\partial s} \varphi(s, z) \mathrm{d} z \mathrm{~d} s\right| & \left|\int_{0} \int_{\Omega^{\varepsilon}} \widetilde{\mathcal{A}}^{\varepsilon} w_{\varepsilon}(s, z) \varphi(s, z) \mathrm{d} z \mathrm{~d} s\right| \\
+\sqrt{\varepsilon}\left|\int_{0} \int_{\Omega^{\varepsilon}} \widetilde{B}^{\varepsilon}(z) \cdot \nabla w_{\varepsilon}(s, z) \varphi(s, z) \mathrm{d} z \mathrm{~d} s\right| & +\left|\frac{1}{2} \int_{0}^{S} \int_{\Omega^{\varepsilon}} \widetilde{\Sigma}^{\varepsilon}(z) w_{\varepsilon}(s, z) \varphi(s, z) \mathrm{d} z \mathrm{~d} s\right| \\
& +\left|\int_{0}^{S} \int_{\Omega^{\varepsilon}} \widetilde{\sigma}^{\varepsilon}(z) \frac{\lambda_{\infty}(\sqrt{\varepsilon} z)-\lambda_{\infty}}{2 \varepsilon} w_{\varepsilon}(s, z) \varphi(s, z) \mathrm{d} z \mathrm{~d} s\right| .
\end{aligned}
$$

By (4.12) and the hypotheses of the coefficients, we get

$$
\left|\int_{0}^{S} \int_{\Omega^{\varepsilon}} \widetilde{\mathcal{A}}^{\varepsilon} w_{\varepsilon}(s, z) \varphi(s, z) \mathrm{d} z \mathrm{~d} s\right| \leq c\left\|\nabla w_{\varepsilon}\right\|_{L^{2}\left((0, S) \times \Omega^{\varepsilon}\right)}\|\nabla \varphi\|_{L^{2}\left((0, S) \times \Omega^{\varepsilon}\right)}+c \sqrt{\varepsilon}\left\|\nabla w_{\varepsilon}\right\|_{L^{2}\left((0, S) \times \Omega^{\varepsilon}\right)}\|\varphi\|_{L^{2}\left((0, S) \times \Omega^{\varepsilon}\right)} .
$$

Since the vector $\widetilde{B}^{\varepsilon}$ and $\widetilde{\Sigma}^{\varepsilon}$ are uniformly bounded, Cauchy-Schwartz inequality yields

$$
\begin{gathered}
\left|\int_{0}^{S} \int_{\Omega^{\varepsilon}} \widetilde{B}^{\varepsilon}(z) \cdot \nabla w_{\varepsilon}(s, z) \varphi(s, z) \mathrm{d} z \mathrm{~d} s\right| \leq c\left\|\nabla w_{\varepsilon}\right\|_{L^{2}\left((0, S) \times \Omega^{\varepsilon}\right)}\|\varphi\|_{L^{2}\left((0, S) \times \Omega^{\varepsilon}\right)}, \\
\left|\frac{1}{2} \int_{0}^{S} \int_{\Omega^{\varepsilon}} \widetilde{\Sigma}^{\varepsilon}(z) w_{\varepsilon}(s, z) \varphi(s, z) \mathrm{d} z \mathrm{~d} s\right| \leq c\left\|w_{\varepsilon}\right\|_{L^{2}\left((0, S) \times \Omega^{\varepsilon}\right)}\|\varphi\|_{L^{2}\left((0, S) \times \Omega^{\varepsilon}\right)} .
\end{gathered}
$$

On the other hand, the hypothesis H2 implies (4.13) and, therefore,

$$
\left|\int_{0}^{S} \int_{\Omega^{\varepsilon}} \tilde{\sigma}^{\varepsilon}(z) \frac{\lambda_{\infty}(\sqrt{\varepsilon} z)-\lambda_{\infty}}{2 \varepsilon} w_{\varepsilon}(s, z) \varphi(s, z) \mathrm{d} z \mathrm{~d} s\right| \leq c\left\|w_{\varepsilon}\right\|_{L^{2}\left((0, S) ; L^{2}\left(\Omega^{\varepsilon} ;|z|^{2}\right)\right)}\|\varphi\|_{L^{2}\left((0, S) ; L^{2}\left(\Omega^{\varepsilon} ;|z|^{2}\right)\right)}
$$

Thus, using the fact that $C>\sigma^{\varepsilon}(z)>c>0$ and the estimate (4.9), we conclude the proof of (4.10). 
As a consequence of the previous a priori estimates and using the Aubin-Lions compactness lemma, we have the following compactness result.

Lemma 4.2. Let $w_{\varepsilon}$ be a solution of (4.7). Assume that $w_{\varepsilon}^{0}$ is uniformly bounded in $L^{2}\left(\mathbb{R}^{N}\right)$. Then, up to as subsequence, there exists a limit $w(s, z) \in L^{\infty}\left(\mathbb{R}^{+} ; L^{2}\left(\mathbb{R}^{N}\right)\right) \cap L^{2}\left(\mathbb{R}^{+} ; H^{1}\left(\mathbb{R}^{N}\right)\right) \cap L^{2}\left(\mathbb{R}^{+} ; L^{2}\left(\mathbb{R}^{N} ;|z|^{2}\right)\right)$ such that

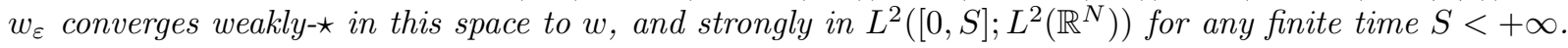

Proof. By the a priori estimate (4.9), up to a subsequence, $w_{\varepsilon}$ converges weakly- $\star$ to a limit $w$ in the space $L^{\infty}\left(\mathbb{R}^{+} ; L^{2}\left(\mathbb{R}^{N}\right)\right) \cap L^{2}\left(\mathbb{R}^{+} ; H^{1}\left(\mathbb{R}^{N}\right)\right) \cap L^{2}\left(\mathbb{R}^{+} ; L^{2}\left(\mathbb{R}^{N} ;|z|^{2}\right)\right)$. On the other hand, it is well-known that $H^{1}\left(\mathbb{R}^{N}\right) \cap$ $L^{2}\left(\mathbb{R}^{N} ;|z|^{2}\right)$ is included in $L^{2}\left(\mathbb{R}^{N}\right)$ with compact embedding. In view of estimate (4.10), the time derivative $\frac{\partial w^{\varepsilon}}{\partial t}$ is uniformly bounded in the dual space of the Hilbert space $L^{2}\left([0, S] ; H^{1}\left(\mathbb{R}^{N}\right)\right) \cap L^{2}\left([0, S] ; L^{2}\left(\mathbb{R}^{N} ;|z|^{2}\right)\right)$. Then, using the classical Aubin-Lions compactness lemma (see [19]), the sequence $w_{\varepsilon}$ is relatively compact in $L^{2}\left([0, S] ; L^{2}\left(\mathbb{R}^{N}\right)\right)$ for any finite time $S$.

We now briefly recall the notion of two-scale convergence (see $[1,16]$ ). Below, we denote by $\delta>0$ the period which is going to 0 , since we shall apply this result not for $\varepsilon$ but rather for $\sqrt{\varepsilon}$.

Proposition 4.3. Let $\delta>0$ denote a sequence of positive reals going to zero. Let $w_{\delta}$ be a bounded sequence in $L^{2}(\Omega)$. There exist a subsequence, still denoted by $\delta$, and a limit $w(x, y) \in L^{2}\left(\Omega ; L^{2}\left(\mathbb{T}^{N}\right)\right)$ such that $w_{\delta}$ two-scale converges to $w$ in the sense that

$$
\lim _{\delta \rightarrow 0} \int_{\Omega} w_{\delta}(x) \phi(x, x / \delta) \mathrm{d} x=\int_{\Omega} \int_{\mathbb{T}^{N}} w(x, y) \phi(x, y) \mathrm{d} x \mathrm{~d} y
$$

for all functions $\phi(x, y) \in L^{2}\left(\Omega ; C\left(\mathbb{T}^{N}\right)\right)$.

Furthermore, if $w_{\delta}$ is a bounded sequence that converges weakly to a limit $w$ in $H^{1}(\Omega)$. Then, wo two-scale converges to $w$, and there exists a function $w_{1}(x, y) \in L^{2}\left(\Omega ; H^{1}\left(\mathbb{T}^{N}\right)\right)$ such that, up to a sequence, $\nabla w_{\delta}$ two-scale converges to $\nabla_{x} w(x)+\nabla_{y} w_{1}(x, y)$.

Proof of Theorem 3.1. Equation (4.7) is a combined problem of homogenization and singular perturbations: the coefficients are oscillating with a period $\sqrt{\varepsilon}$, and they concentrate to 0 with respect to their first macroscopic argument. Therefore, we expect that the limit problem of (4.7) is precisely the homogenized problem (3.4). To prove this statement and study the asymptotic behavior of $(4.7)$, we follow the methodology of $[7,8]$.

We multiply (4.7) by a test function $\phi_{\varepsilon}(s, z)=\phi(s, z)+\sqrt{\varepsilon} \phi_{1}(s, z, z / \sqrt{\varepsilon})$ where $\phi(s, z)$ and $\phi_{1}(s, z, y)$ are smooth test functions defined on $\mathbb{R}^{+} \times \mathbb{R}^{N}$ and $\mathbb{R}^{+} \times \mathbb{R}^{N} \times \mathbb{T}^{N}$, respectively, and with compact support in $\mathbb{R}^{+} \times \mathbb{R}^{N}$. For sufficiently small $\varepsilon$, the support of $\phi_{\varepsilon}$ is included in $\Omega^{\varepsilon}$ for all times. Integrating by parts yields

$$
\begin{array}{r}
\int_{0}^{\infty} \int_{\mathbb{R}^{N}} \widetilde{\sigma}^{\varepsilon}(z) w_{\varepsilon} \frac{\partial \phi_{\varepsilon}}{\partial s} \mathrm{~d} z \mathrm{~d} s+\int_{0}^{\infty} \int_{\mathbb{R}^{N}} \widetilde{\mathcal{A}}^{\varepsilon} w_{\varepsilon} \phi_{\varepsilon} \mathrm{d} z \mathrm{~d} s+\int_{0}^{\infty} \int_{\mathbb{R}^{N}} \widetilde{\sigma}^{\varepsilon} \frac{\lambda_{\infty}(\sqrt{\varepsilon} z)-\lambda_{\infty}}{\varepsilon} w_{\varepsilon} \phi_{\varepsilon} \mathrm{d} z \mathrm{~d} s \\
+\sqrt{\varepsilon} \int_{0}^{\infty} \int_{\mathbb{R}^{N}} \widetilde{B}^{\varepsilon}(z) \cdot \nabla w_{\varepsilon} \phi_{\varepsilon} \mathrm{d} z \mathrm{~d} s+\int_{0}^{\infty} \int_{\mathbb{R}^{N}}^{\infty} \widetilde{\Sigma}^{\varepsilon}(z) w_{\varepsilon} \phi_{\varepsilon} \mathrm{d} z \mathrm{~d} s=0 .
\end{array}
$$

In view of the a priori estimate (4.9), we get

$$
\sqrt{\varepsilon} \int_{0}^{\infty} \int_{\mathbb{R}^{N}} \widetilde{B}^{\varepsilon}(z) \cdot \nabla w_{\varepsilon}(s, z) \phi_{\varepsilon}(s, z) \mathrm{d} z \mathrm{~d} s \rightarrow 0
$$


and, since $\Sigma^{\varepsilon}=\Sigma_{0}^{\varepsilon}+\varepsilon \Sigma_{1}^{\varepsilon}$,

$$
\varepsilon \int_{0}^{\infty} \int_{\mathbb{R}^{N}} \widetilde{\Sigma}_{1}^{\varepsilon}(z) w_{\varepsilon}(s, z) \phi_{\varepsilon}(s, z) \mathrm{d} z \mathrm{~d} s \rightarrow 0
$$

On the compact and fixed space support of $\phi_{\varepsilon}$, the values of the smooth coefficients at points $(\sqrt{\varepsilon} z, z / \sqrt{\varepsilon})$ are uniformly close to their values at $(0, z / \sqrt{\varepsilon})$. Thus, since by virtue of Lemma 4.2 any average in time of $w_{\varepsilon}$ converges strongly to the same average of $w$ in $L^{2}\left(\mathbb{R}^{N}\right)$, we get

$$
\begin{aligned}
& \int_{0}^{\infty} \int_{\mathbb{R}^{N}} \widetilde{\sigma}^{\varepsilon}(z) w_{\varepsilon} \frac{\partial \phi_{\varepsilon}}{\partial s} \mathrm{~d} z \mathrm{~d} s \rightarrow \int_{0}^{\infty} \int_{\mathbb{R}^{N}} w \frac{\partial \phi}{\partial s} \mathrm{~d} z \mathrm{~d} s \\
& \int_{0}^{\infty} \int_{\mathbb{R}^{N}} \widetilde{\Sigma}_{0}^{\varepsilon}(z) w_{\varepsilon}(s, z) \phi_{\varepsilon}(s, z) \mathrm{d} z \mathrm{~d} s \rightarrow \int_{0}^{\infty} \int_{\mathbb{R}^{N}} d^{\text {eff }} w(s, z) \phi(s, z) \mathrm{d} z \mathrm{~d} s .
\end{aligned}
$$

By assumptions H2 we get

$$
\int_{0}^{\infty} \int_{\mathbb{R}^{N}} \tilde{\sigma}^{\varepsilon} \frac{\lambda_{\infty}(\sqrt{\varepsilon} z)-\lambda_{\infty}}{\varepsilon} w_{\varepsilon} \phi_{\varepsilon} \mathrm{d} z \mathrm{~d} s=\int_{0}^{\infty} \int_{\mathbb{R}^{N}} \tilde{\sigma}^{\varepsilon} \Lambda_{i j} z_{i} z_{j} w_{\varepsilon} \phi \mathrm{d} z \mathrm{~d} s \quad+o(1)
$$

and the same argument of strong convergence in $L^{2}\left(\mathbb{R}^{N}\right)$ of time averages of $w_{\varepsilon}$ implies

$$
\int_{0}^{\infty} \int_{\mathbb{R}^{N}} \tilde{\sigma}^{\varepsilon} \frac{\lambda_{\infty}(\sqrt{\varepsilon} z)-\lambda_{\infty}}{\varepsilon} w_{\varepsilon} \phi_{\varepsilon} \mathrm{d} z \mathrm{~d} s \rightarrow \int_{0}^{\infty} \int_{\mathbb{R}^{N}} \Lambda_{i j} z_{i} z_{j} w \phi \mathrm{d} z \mathrm{~d} s
$$

Finally, passing to the two-scale limit (see Prop. 4.3), there exists a function $w_{1}(s, z, y)$ defined in $\mathbb{R}^{+} \times \mathbb{R}^{N} \times \mathbb{T}^{N}$, such that

$$
\begin{array}{r}
\lim _{\varepsilon \rightarrow 0} \int_{0}^{\infty} \int_{\mathbb{R}^{N}} \widetilde{\mathcal{A}}^{\varepsilon} w_{\varepsilon} \phi_{\varepsilon} \mathrm{d} z \mathrm{~d} s=\int_{0}^{\infty} \int_{\mathbb{R}^{N}} \int_{\mathbb{T}} \psi_{\infty} \psi_{\infty}^{*} a_{i j}(0, y)\left(\frac{\partial w}{\partial x_{j}}+\frac{\partial w_{1}}{\partial y_{j}}\right)\left(\frac{\partial \phi}{\partial x_{i}}+\frac{\partial \phi_{1}}{\partial y_{i}}\right) \mathrm{d} y \mathrm{~d} z \mathrm{~d} s \\
+\int_{0}^{\infty} \int_{\mathbb{R}^{N}} \int_{\mathbb{T}} d_{i j}(0, y)\left(\frac{\partial w}{\partial x_{j}}+\frac{\partial w_{1}}{\partial y_{j}}\right)\left(\frac{\partial \phi}{\partial x_{i}}+\frac{\partial \phi_{1}}{\partial y_{i}}\right) \mathrm{d} y \mathrm{~d} z \mathrm{~d} s
\end{array}
$$

where the skew-symmetric matrix $D=\left\{d_{i j}\right\}$ is defined by (4.11). Regrouping all terms we obtain a limit variational formulation for the couple $\left(w, w_{1}\right)$. In order to eliminate $w_{1}$, we take $\phi \equiv 0$ and, integrating by parts, we obtain that $w_{1}$ satisfies

$$
-\frac{\partial}{\partial y_{i}}\left[\psi_{\infty} \psi_{\infty}^{*} a_{i j}(0, y)\left(\frac{\partial w}{\partial x_{j}}+\frac{\partial w_{1}}{\partial y_{j}}\right)\right]-\frac{\partial}{\partial y_{i}}\left[d_{i j}(0, y)\left(\frac{\partial w}{\partial x_{j}}+\frac{\partial w_{1}}{\partial y_{j}}\right)\right]=0 \quad \text { in } \mathbb{R}^{+} \times \mathbb{R}^{N} \times \mathbb{T}
$$

Since $\operatorname{div}_{y} D=\beta$, we get that $w_{1}$ is the weak solution of

$$
-\operatorname{div}_{y}\left(\psi_{\infty} \psi_{\infty}^{*} A(0, y)\left(\nabla_{x} w+\nabla_{y} w_{1}\right)\right)+\beta(0, y)\left(\nabla_{x} w+\nabla_{y} w_{1}\right)=0 \quad \text { in } \mathbb{R}^{+} \times \mathbb{R}^{N} \times \mathbb{T}
$$


By linearity, we obtain a solution of (4.16) by taking

$$
w_{1}(s, z, y)=\frac{\partial w}{\partial x_{i}}(s, z) \chi_{i}(y)
$$

where the functions $\chi_{i}$ satisfy (3.7). On the other hand, returning to the weak formulation and considering $\phi_{1} \equiv 0$, it is immediate with the above expression of $w_{1}$ that

$$
\begin{array}{r}
\lim _{\varepsilon \rightarrow 0} \int_{0}^{\infty} \int_{\mathbb{R}^{N}} \widetilde{\mathcal{A}}^{\varepsilon} w_{\varepsilon} \cdot \phi \mathrm{d} z \mathrm{~d} s=\int_{\mathbb{T}} \psi_{\infty} \psi_{\infty}^{*}(0, y)\left(a_{i j}(0, y)+a_{i k}(0, y) \frac{\partial \chi_{j}}{\partial y_{k}}\right) \mathrm{d} y \int_{0}^{\infty} \int_{\mathbb{R}^{N}} \frac{\partial w}{\partial x_{j}} \frac{\partial \phi}{\partial x_{i}} \mathrm{~d} z \mathrm{~d} s \\
+\int_{\mathbb{T}}\left(d_{i j}(0, y)+d_{i k}(0, y) \frac{\partial \chi_{j}}{\partial y_{k}}\right) \mathrm{d} y \int_{0}^{\infty} \frac{\partial w}{\partial x_{j}} \frac{\partial \phi}{\partial x_{i}} \mathrm{~d} z \mathrm{~d} s
\end{array}
$$

Since the homogenized coefficients $\left\{a_{i j}^{\text {eff }}\right\}$, defined by (3.6), are constant and $D=\left\{d_{i j}\right\}$ is skew-symmetric

$$
a_{i j}^{\mathrm{eff}}=\int_{\mathbb{T}}\left(\psi_{\infty} \psi_{\infty}^{*} A(0, y)+D(0, y)\right) \nabla\left(\chi_{j}+y_{j}\right) \cdot \nabla\left(\chi_{i}+y_{i}\right) \mathrm{d} y .
$$

Now, by definition of $D=\left\{d_{i j}\right\}$ in (4.11), we get for any $i$

$$
\int_{\mathbb{T}}\left(\psi_{\infty} \psi_{\infty}^{*} A(0, y)+D(0, y)\right) \nabla\left(\chi_{j}+y_{j}\right) \cdot \nabla \chi_{i} \mathrm{~d} y=0
$$

since $\chi_{j}$ is solution of (3.7). Therefore, we obtain

$$
\lim _{\varepsilon \rightarrow 0} \int_{0}^{\infty} \int_{\mathbb{R}^{N}} \widetilde{\mathcal{A}}^{\varepsilon} w_{\varepsilon} \cdot \phi \mathrm{d} z \mathrm{~d} s=-a_{i j}^{\text {eff }} \int_{0}^{\infty} \int_{\mathbb{R}^{N}} \frac{\partial^{2} w}{\partial x_{j} \partial x_{i}} \phi \mathrm{d} z \mathrm{~d} s .
$$

Finally, by (4.1) and (4.6), we get

$$
w_{\varepsilon}(0, z)=\frac{u_{\varepsilon}^{0}(\sqrt{\varepsilon} z)}{\psi_{\infty}(\sqrt{\varepsilon} z, z / \sqrt{\varepsilon})},
$$

and, by the hypothesis $(3.2)$ on the initial data $u_{\varepsilon}^{0}$, we conclude $w_{\varepsilon}(0, z)=u_{0}(z)$. Summing up all the limits, we conclude that $w$ is the unique weak solution of (3.4). By uniqueness of the limit, the entire sequence $w_{\varepsilon}$ converges.

\section{Eigenvalue problem}

This section is devoted to the proof of Theorem 3.5 concerning the behavior of the first eigenvalue of the spectral problem (3.8). As already said in Remark 3.6, the homogenized spectral problem (3.10) has a countable infinite number of eigenvalues of finite multiplicity. Indeed, the corresponding Green operator acts from $L^{2}\left(\mathbb{R}^{N}\right)$ into $H^{1}\left(\mathbb{R}^{N}\right) \cap L^{2}\left(\mathbb{R}^{N} ;|z|^{2}\right)$, and thus is self-adjoint and compact in $L^{2}\left(\mathbb{R}^{N}\right)$. Furthermore, by the Krein-Rutman theorem, the first eigenvalue $\mu_{1}$ is of multiplicity one and the corresponding eigenfunction $q_{1} \in H^{1}\left(\mathbb{R}^{N}\right) \cap$ $L^{2}\left(\mathbb{R}^{N} ;|z|^{2}\right)$ can be chosen positive.

As in the previous section, we introduce a new unknown

$$
p_{\varepsilon}(x)=\frac{p_{1}^{\varepsilon}(x)}{\psi_{\infty}(x, x / \varepsilon)}
$$


After a little algebra, $p_{\varepsilon}$ is solution of

$$
\left\{\begin{array}{l}
-\varepsilon \operatorname{div}\left(\alpha^{\varepsilon} \nabla p_{\varepsilon}\right)+\beta^{\varepsilon} \cdot \nabla p_{\varepsilon}+\sigma^{\varepsilon} \frac{\lambda_{\infty}(x)-\lambda_{\infty}}{\varepsilon} p_{\varepsilon}+\varepsilon B^{\varepsilon} \cdot \nabla p_{\varepsilon}+\Sigma^{\varepsilon} p_{\varepsilon}=\mu^{\varepsilon} \sigma^{\varepsilon} p_{\varepsilon} \quad \text { in } \Omega \\
p_{\varepsilon}=0 \quad \text { on } \partial \Omega
\end{array}\right.
$$

where $\mu^{\varepsilon}=\varepsilon^{-1}\left(\lambda_{1}^{\varepsilon}-\lambda_{\infty}\right)$. The coefficients $\sigma^{\varepsilon}, \alpha^{\varepsilon}, \beta^{\varepsilon}, B^{\varepsilon}$ and $\Sigma^{\varepsilon}$ are defined in (4.3) and (4.4). In order to eliminate the $\varepsilon$ scaling in front of the second-order operator in (5.2), we rescale the space variable by introducing

$$
z=\frac{x}{\sqrt{\varepsilon}} \in \Omega^{\varepsilon}=\varepsilon^{-1 / 2} \Omega \text { and } q_{\varepsilon}(z)=p_{\varepsilon}(x) .
$$

This yields

$$
\left\{\begin{array}{l}
\widetilde{\mathcal{A}}^{\varepsilon} q_{\varepsilon}+\widetilde{\sigma}^{\varepsilon} \frac{\lambda_{\infty}(\sqrt{\varepsilon} z)-\lambda_{\infty}}{\varepsilon} q_{\varepsilon}+\sqrt{\varepsilon} \widetilde{B}^{\varepsilon} \cdot \nabla q_{\varepsilon}+\widetilde{\Sigma}^{\varepsilon} q_{\varepsilon}=\mu^{\varepsilon} \widetilde{\sigma}^{\varepsilon} q_{\varepsilon} \quad \text { in } \Omega^{\varepsilon} \\
q_{\varepsilon}=0 \quad \text { on } \partial \Omega^{\varepsilon},
\end{array}\right.
$$

where the operator $\widetilde{\mathcal{A}}^{\varepsilon}$ is defined by (4.12) and the other coefficients are as in the previous section. Equation (5.3) is a combined problem of homogenization and singular perturbations: the coefficients are microscopically oscillating with period $\sqrt{\varepsilon}$, and they macroscopically concentrate at the origin $z=0$. The domain $\Omega^{\varepsilon}$ is converging to $\mathbb{R}^{N}$. Therefore, we expect that the limit problem of (5.3) is precisely the homogenized problem (3.10).

To prove this statement, we follow the methodology of [7]. We introduce the corresponding Green operator $S^{\varepsilon}: L^{2}\left(\Omega^{\varepsilon}\right) \rightarrow L^{2}\left(\Omega^{\varepsilon}\right)$ such that $S^{\varepsilon}(f)=u^{\varepsilon}$ where $u^{\varepsilon}$ is the unique solution in $H_{0}^{1}\left(\Omega^{\varepsilon}\right)$ of

$$
\left\{\begin{array}{l}
\widetilde{\mathcal{A}}^{\varepsilon} u^{\varepsilon}+\widetilde{\sigma}^{\varepsilon} \frac{\lambda_{\infty}(\sqrt{\varepsilon} z)-\lambda_{\infty}}{\varepsilon} u^{\varepsilon}+\sqrt{\varepsilon} \widetilde{B}^{\varepsilon} \cdot \nabla u^{\varepsilon}+\widetilde{\Sigma}^{\varepsilon} u^{\varepsilon}=\widetilde{\sigma}^{\varepsilon} f \quad \text { in } \Omega^{\varepsilon}, \\
u^{\varepsilon}=0 \quad \text { on } \partial \Omega^{\varepsilon}
\end{array}\right.
$$

We shall assume without loss of generality that $\widetilde{\Sigma}^{\varepsilon}$ is positive (indeed, a simple shift in the spectrum amounts to add a multiple of $\widetilde{\sigma}^{\varepsilon}$, which is positive, to $\widetilde{\Sigma}^{\varepsilon}$. In the sequel we consider that $S^{\varepsilon}$ is an operator defined in $L^{2}\left(\mathbb{R}^{N}\right)$ by simply taking $f$ as the restriction to $\Omega^{\varepsilon}$ of a function of $L^{2}\left(\mathbb{R}^{N}\right)$ and extending by zero outside $\Omega^{\varepsilon}$ the solution $u^{\varepsilon}=S^{\varepsilon}(f)$. The homogenization of (5.4) is similar to what we did in the previous section. We introduce the limit Green operator $S: L^{2}\left(\mathbb{R}^{N}\right) \rightarrow L^{2}\left(\mathbb{R}^{N}\right)$ such that $S(f)=u$ where $u$ is the unique solution in $H_{0}^{1}\left(\mathbb{R}^{N}\right)$ of

$$
-\operatorname{div}\left(A^{\mathrm{eff}} \nabla u\right)+\left(\Lambda_{i j} z_{i} z_{j}+d^{\mathrm{eff}}\right) u=f \quad \text { in } \mathbb{R}^{N},
$$

where the coefficient $d^{\text {eff }}$ and the matrix $A^{\text {eff }}$ are defined by (3.5)-(3.6). Then, we have the following convergence result (see Lem. 4.4 of [7])

Lemma 5.1. The sequence of operators $S^{\varepsilon}$ compactly converges to the limit operator $S$ in the sense that

$$
\begin{aligned}
& \text { (i) for any } f \in L^{2}\left(\mathbb{R}^{N}\right), \quad \lim _{\varepsilon \rightarrow 0}\left\|S^{\varepsilon}(f)-S(f)\right\|_{L^{2}\left(\mathbb{R}^{N}\right)}=0, \\
& \text { (ii) } \text { the set }\left\{S^{\varepsilon}(f):\|f\|_{L^{2}\left(\mathbb{R}^{N}\right)} \leq 1, \varepsilon \geq 0\right\} \text { is sequentially compact. }
\end{aligned}
$$

Proof. The proof is quite classical (see e.g., $[3,4,7]$ ) for similar examples), so we simply indicate the main ingredients. First, we multiply $(5.4)$ by $u^{\varepsilon}$ and integrate by parts to obtain a priori estimate. Since by assumptions $\mathrm{H} 1$ and $\mathrm{H} 2$ we have (4.13), we get

$$
\left\|\nabla u^{\varepsilon}\right\|_{L^{2}\left(\mathbb{R}^{N}\right)}+\left\||z| u^{\varepsilon}\right\|_{L^{2}\left(\mathbb{R}^{N}\right)} \leq C\|f\|_{L^{2}\left(\mathbb{R}^{N}\right)} .
$$

This implies that the sequence $u^{\varepsilon}$ is pre-compact in $H^{1}\left(\mathbb{R}^{N}\right)$-weak and pre-compact in $L^{2}\left(\mathbb{R}^{N}\right)$-strong. Second, we pass to the limit in (5.4) using the two-scale convergence (see Prop. 4.3). We multiply (5.4) by a test function 
$\phi(z)+\sqrt{\varepsilon} \phi_{1}(z, z / \sqrt{\varepsilon})$ where $\phi$ and $\phi_{1}$ are smooth test functions defined on $\mathbb{R}^{N}$ and $\mathbb{R}^{N} \times \mathbb{T}^{N}$, respectively, and with compact support in $\mathbb{R}^{N}$ and periodic with respect to the second variable $y=z / \sqrt{\varepsilon}$. Since this test function has compact support (fixed with respect to $\varepsilon$ ), the effect of the non-periodic modulation in the coefficients is negligible. Now, this is a standard matter in the theory of two-scale convergence (see the previous section) to deduce that any converging subsequence of $u^{\varepsilon}$ converges weakly in $H^{1}\left(\mathbb{R}^{N}\right)$ to $u$ which is the unique solution of (5.5). The homogenized coefficients in (5.5) are thus obtained by considering the cell problems with the frozen macroscopic variable $x=0$. By uniqueness of the limit, the entire sequence $u^{\varepsilon}$ converges. Furthermore, estimate (5.6) shows that $u^{\varepsilon}$ does also converge strongly in $L^{2}\left(\mathbb{R}^{N}\right)$. This proves statement $(i)$ of the lemma. To prove statement $(i i)$ we simply remark that estimate (5.6) as well as the strong $L^{2}\left(\mathbb{R}^{N}\right)$ convergence of $u^{\varepsilon}$ is still valid if the right hand side $f$ is replaced by a bounded sequence $f^{\varepsilon}$ in $L^{2}\left(\mathbb{R}^{N}\right)$. This shows that $S^{\varepsilon}$ compactly converges to $\mathrm{S}$.

To finish the proof of Theorem 3.5, we now recall a classical result in operator theory that can be found, for example, in [9].

Theorem 5.2. Let $X$ be a Banach space and $\mathcal{L}(X)$ be the set of bounded linear operators in $X$. Let $\left\{T_{n}\right\}_{n \in \mathbb{N}}$ be a sequence of operators in $\mathcal{L}(X)$ converging compactly to $T$ in the sense that:

- for all $x \in X, T_{n} x \rightarrow T x$ as $n \rightarrow \infty$,

- for any bounded sequence $\left\{x_{n}\right\}_{n \in \mathbb{N}}$ with $\left\|x_{n}\right\| \leq 1$, the sequence $\left\{\left(T-T_{n}\right) x_{n}\right\}_{n \in \mathbb{N}}$ is relatively compact in $X$.

Let $\sigma(T)$ and $\sigma\left(T_{n}\right)$ be the spectra of $T$ and $T_{n}$, respectively. Let $\lambda$ be an isolated eigenvalue of $T$ of finite multiplicity and let $\Gamma$ be a closed Jordan curve in the complex plane around $\lambda$ and isolating $\lambda$ such that the domain $\Delta$ enclosed by $\Gamma$ contain no other point of the spectrum $\sigma(T)$ than $\lambda$. Then, $\sigma\left(T_{n}\right) \cap \Delta$ contains a number of eigenvalues equal to the multiplicity of $\lambda$ provided $n$ is large enough.

Moreover, if $\lambda_{n}$ is a sequence of eigenvalues of $T_{n}$ converging to $\lambda$, and $u_{n}$ is a sequence of normalized associated eigenvectors, then, up to a subsequence, $u_{n}$ converges to a limit $u$ in $X$ which is an eigenvector of $T$ associated with $\lambda$.

By Lemma 5.1, $S^{\varepsilon}$ converges compactly to $S$, and we can apply Theorem 5.2 to conclude that the spectrum of $S^{\varepsilon}$ converges to that of $S$. With this we conclude the proof of Theorem 3.5.

Acknowledgements. This work was partly done during the stay of R. Orive at École Polytechnique in September 2004. R. Orive has been partially supported by grant BFM2005-00714 of the MEC (SPAIN). This author gratefully acknowledges the warm hospitality of the Centre de Mathématiques Appliquées at École Polytechnique received during his stays.

\section{REFERENCES}

[1] G. Allaire, Homogenization and two-scale convergence. SIAM J. Math. Anal. 23 (1992) 1482-1518.

[2] G. Allaire, Dispersive limits in the homogenization of the wave equation. Annales de la Faculté des Sciences de Toulouse XII (2003) 415-431.

[3] G. Allaire and Y. Capdeboscq, Homogenization of a spectral problem in neutronic multigroup diffusion. Comput. Methods Appl. Mech. Engrg. 187 (2000) 91-117.

[4] G. Allaire and C. Conca, Bloch wave homogenization and spectral asymptotic analysis. J. Math. Pures Appl. 77 (1998) 153-208.

[5] G. Allaire and F. Malige, Analyse asymptotique spectrale d'un probléme de diffusion neutronique. C. R. Acad. Sci. Paris Sér. I 324 (1997) 939-944.

[6] G. Allaire and R. Orive, On the band gap structure of Hill's equation. J. Math. Anal. Appl. 306 (2005) 462-480.

[7] G. Allaire and A. Piatnitski, Uniform spectral asymptotics for singularly perturbed locally periodic operator. Comm. Partial Differential Equations 27 (2002) 705-725.

[8] G. Allaire, Y. Capdeboscq, A. Piatnitski, V. Siess and M. Vanninathan, Homogenization of periodic systems with large potentials. Arch. Rational Mech. Anal. 174 (2004) 179-220. 
[9] P.H. Anselone, Collectively compact operator approximation theory and applications to integral equations. Series in Automatic Computation, Prentice-Hall, Inc., Englewood Cliffs, New Jersey (1971).

[10] A. Benchérif-Madani and É. Pardoux, Locally periodic homogenization. Asymptot. Anal. 39 (2004) 263-279.

[11] A. Benchérif-Madani and É. Pardoux, Homogenization of a diffusion with locally periodic coefficients. Séminaire de Probabilités XXXVIII Lect. Notes Math. 1857 (2005) 363-392.

[12] A. Bensoussan, J.L. Lions and G. Papanicolaou, Asymptotic Analysis for Periodic Structures. North-Holland, Amsterdam (1978).

[13] Y. Capdeboscq, Homogenization of a diffusion equation with drift. C. R. Acad. Sci. Paris Sér. I 327 (1998) $807-812$.

[14] Y. Capdeboscq, Homogenization of a neutronic critical diffusion problem with drift. Proc. Roy. Soc. Edinburgh Sect. A 132 (2002) 567-594.

[15] P. Donato and A. Piatnitski, Averaging of nonstationary parabolic operators with large lower order terms. (2005) (in preparation).

[16] G. Nguetseng, A general convergence result for a functional related to the theory of homogenization. SIAM J. Math. Anal. 20 (1989) 608-623.

[17] A. Piatnitski, Asymptotic behaviour of the ground state of singularly perturbed elliptic equations. Commun. Math. Phys. 197 (1998) 527-551.

[18] A. Piatnitski, Ground State Asymptotics for Singularly Perturbed Elliptic Problem with Locally Periodic Microstructure. Preprint (2006).

[19] J. Simon, Compact sets in the space $L^{p}(0, T ; B)$. Ann. Mat. Pura Appl. 146 (1987) 65-96.

[20] S. Sivaji Ganesh and M. Vanninathan, Bloch wave homogenization of scalar elliptic operators. Asymptotic Anal. 39 (2004) $15-44$.

[21] M. Vanninathan, Homogenization of eigenvalue problems in perforated domains. Proc. Indian Acad. Sci. Math. Sci. 90 (1981) $239-271$. 